\title{
Pratiques
}

Linguistique, littérature, didactique

$169-170 \mid 2016$

Enseignement/apprentissage de la langue, des textes et des discours. 40\&nbspans de Pratiques

\section{La " grammaire de phrase » dans les textes officiels depuis le Plan de rénovation}

Syntax Teaching in Official Instructions since the "Plan de renovation"

\section{Bernard Combettes}

\section{(2) OpenEdition}

Journals

Édition électronique

URL : http://journals.openedition.org/pratiques/3082

DOI : $10.4000 /$ pratiques.3082

ISSN : 2425-2042

Éditeur

Centre de recherche sur les médiations (CREM)

Référence électronique

Bernard Combettes, « La « grammaire de phrase » dans les textes officiels depuis le Plan de

rénovation », Pratiques [En ligne], 169-170 | 2016, mis en ligne le 30 juin 2016, consulté le 19 avril 2019

URL : http://journals.openedition.org/pratiques/3082 ; DOI : 10.4000/pratiques.3082

Ce document a été généré automatiquement le 19 avril 2019

(C) Tous droits réservés 


\title{
La « grammaire de phrase » dans les textes officiels depuis le Plan de rénovation
}

\author{
Syntax Teaching in Official Instructions since the "Plan de renovation"
}

\section{Bernard Combettes}

1 Les nouveaux programmes d'enseignement pour l'école élémentaire et pour le collège, de parution récente, en novembre 2015, nous semblent présenter des aspects particulièrement novateurs, qu'il s'agisse des contenus ou de la méthodologie qu'ils proposent pour l'étude de la langue. Ces aspects, que nous évoquerons à la fin de cet article, ne peuvent être bien perçus que s'ils sont replacés dans une dimension historique. C'est ce parcours dans les Instructions officielles successives publiées depuis plus d'une trentaine d'années que nous essayerons de réaliser ici, en limitant nos observations à un point particulier des programmes de français, celui de la syntaxe ou, comme le formulent certains textes, celui de la " grammaire de phrase ».

\section{Le Plan de rénovation}

Dans une perspective historique, il convient de partir de la rupture créée par le «Plan de rénovation " pour l'enseignement du français à l'école primaire, dit « Projet Rouchette ", commencé avant 1968 et publié en 1971. Le point de départ des réflexions et des propositions contenues dans ce document est à rechercher dans une double critique de l'enseignement de la langue et, plus particulièrement, de l'enseignement de la grammaire, tel qu'il était pratiqué à cette époque, à l'école élémentaire comme au collège. Une première critique porte sur les contenus: se trouvent visés la confusion, dans l'analyse grammaticale, des critères formels et des critères sémantiques ainsi que l'absence de prise en compte de la hiérarchisation des structures syntaxiques, dans une progression qui se contente d'aller du mot à la phrase. La deuxième critique, portant sur une démarche pédagogique qui donne la priorité à l'analyse et à la réflexion sur la 
pratique de la langue, condamne « un enseignement grammatical qui, faisant construire les fonctions à partir des natures, passant uniformément de la phrase simple (proposition indépendante) à la phrase complexe, cherche à améliorer l'expression en s'appuyant presque exclusivement sur une réflexion abstraite et non sur le fonctionnement réel de la langue » (Plan de rénovation, p. 4).

Se crée ainsi un ensemble de facteurs qui vont dans le même sens pour faire adopter une démarche conforme aux principes théoriques et aux méthodes de la syntaxe alors en plein renouvèlement. Le recours aux exercices structuraux, explicitement inspirés de la pédagogie des langues étrangères, va de pair avec l'analyse distributionnelle. Alors que la notion de structure n'est pas vraiment exploitée, celle de distribution se présente comme très rigoureuse, ne mêlant pas syntaxe et sémantique. La méthode semble convenir aussi bien à la dimension pratique, dans la mesure où elle implique un maniement des divers constituants, qu'à la grammaire réflexive, la démarche d'observation et de découverte des règles pouvant s'appuyer sur des instruments fiables.

Ce qu'il convient de noter, c'est que l'objet de l'étude, qu'il s'agisse de pratiquer ou d'observer les faits de langue, n'est pas constitué par les notions grammaticales, les natures ou les fonctions, mais bien par le système linguistique, par les structures syntaxiques. Dans les citations suivantes, on relèvera par exemple l'emploi d'expressions comme : "structures syntaxiques", "fonctionnement", "relation", "grandes lois syntaxiques » :

L'entraînement à la communication [...] se renforce et se perfectionne par le maniement, puis par l'observation et l'analyse de structures syntaxiques et de formes lexicales de plus en plus complexes [...] Il s'agit bien plutôt de laisser en marge règles et étiquetages fonctionnels pour faire porter tout l'effort sur la pratique des grandes lois syntaxiques du français. Pour telle «leçon » particulière, le maître aura choisi de travailler sur telle relation grammaticale ; s'étant interrogé sur son fonctionnement, sur les contraintes qu'elle impose et qui font difficulté pour l'enfant, orthographe comprise, il cherchera à construire des exercices de manipulation ». (p. 40)

De ces principes généraux découle, de façon logique, un programme qui n'est pas un programme de notions grammaticales, mais bien un programme de langue, avec des exemples d'énoncés illustrant les structures à travailler, ce qui, on le remarquera, est exceptionnel dans la rédaction de textes officiels, du moins à cette époque. On voit par ailleurs, dans l'extrait suivant, comment les manipulations, les diverses transformations syntaxiques, ne sont pas de simples outils pour l'analyse ou pour la définition des notions, mais bien des moyens de travailler sur l'énoncé et sur les possibilités de la modifier :

Les divers énoncés reposent, d'une part, sur des organisations syntaxiques de base. Il s'agit de les découvrir, c'est-à-dire de mettre en évidence la grammaire des principales phrases simples du français. On considère comme principales phrases simples les types d'énoncés suivants :

- Pierre dort.

2 - La grêle a abîmé le raisin.

[....................]

Ils peuvent tous recevoir un ou plusieurs groupes circonstanciels, dont il paraît vain ici de circonscrire exactement la nuance.

1 - Pierre dort la nuit.

2 - La grêle a abîmé le raisin dans la vigne du haut.

$[$ [.................

Des exercices de maniement pourront permettre de dégager quelques oppositions, par exemple : 
- Circonstanciels / autres groupes.

- Transformables/non transformables.

(La grêle a abîmé le raisin, le raisin a été abîmé par la grêle).

(Le boulanger a donné un croissant à mon frère, mon frère s'est vu donner un croissant par

le boulanger).

[.....................

Dans une telle perspective, l'accent n'est pas mis sur les natures et les fonctions, qui ne sont d'ailleurs pas évoquées, mais sur les formes fondamentales de la phrase, qu'il s'agisse de l'interrogation ou de la négation, qui sont observées à propos de chaque type d'énoncés. La démarche s'oppose donc clairement à l'approche traditionnelle, qui privilégiait la décomposition et l'analyse des unités sans s'attarder sur les structures d'ensemble. La justification de la priorité donnée au travail sur les énoncés est étroitement liée aux finalités de l'enseignement; dans la mesure où il est évident que la communication met en jeu des énoncés et non des unités isolées et que l'observation de la langue a pour but essentiel l'amélioration de l'expression, on comprend la cohérence de cette démarche « ascendante » :

Tous ces exercices de manipulation grammaticale sont évidemment destinés au progrès de la langue orale et écrite de l'enfant. On sera particulièrement attentif à lui faire saisir, par exemple, au détour d'exercices de commutation, les différentes possibilités que lui offre la langue de construire des énoncés. (p. 42).

7 Cette approche est parfaitement illustrée, en 1970, par un ouvrage qui a fait date, Linguistique et enseignement $d u$ français, d'E. Genouvrier et J. Peytard, dans lequel il est constamment affirmé la nécessité de travailler en priorité sur les structures de langue, l'analyse des unités n'étant pas un objectif prioritaire :

Il ne s'agit pas d'étudier tel ou tel type de complément, mais le fonctionnement linguistique d'une structure considérée dans sa totalité. Ce qui nous conduit à

- placer les phrases à verbes impersonnels hors de leur cadre habituel : le sujet ;

- coupler des phrases à verbe être et avoir laissant apercevoir des symétries (j'ai faim / je suis affamé,j'ai un livre / ce livre est à moi) ;

[...]

- introduire les phrases à présentatifs et les phrases segmentées, si riches en français.

Il ne s'agit en aucun cas de se contenter d'une analyse des fonctions au sens habituel du terme, mais d'étudier des procédures linguistiques. Ainsi la structure sujet-verbe-objet sera envisagée essentiellement du point de vue des transformations passives et de leur fonctionnement.

8 Ces suggestions, en accord avec les propositions du Plan de rénovation, ne seront jamais vraiment prises en compte dans les programmes qui se succèderont par la suite; elles restent, encore tout à fait d'actualité aujourd'hui.

\section{Une modernité de surface : les Instructions de 1985}

9 Ce projet de rénovation avait soulevé de nombreuses critiques et les réticences, d'ordre divers et à tous les niveaux, étaient bien trop fortes pour que l'on puisse s'attendre à sa mise en œuvre officielle. Force est en effet de constater qu'il y a bien loin de ces recommandations aux textes officiels qui paraissent à partir de 1976. Le coup d'arrêt est nettement perceptible dès la parution de la circulaire sur la terminologie grammaticale pour l'école élémentaire, qui ne peut manquer d'exprimer, dans son préambule, la méfiance habituelle envers les théories linguistiques et les désaccords des linguistes :

La simplicité et l'uniformité relatives du vocabulaire grammatical utilisé à l'école

élémentaire n'ont pas posé de problème jusqu'au moment où la diffusion de 
théories linguistiques diverses a amené dans les classes l'emploi de terminologies

différentes et parfois concurrentes. donnée à la phrase dans l'ordre des notions, ainsi qu'avec l'introduction de la notion de groupe (groupe nominal et groupe verbal), mais on retrouve la liste habituelle des classes de mots et des fonctions et, surtout, rien n'est dit sur les structures de phrase qui doivent être abordées. On s'en tient à la mention des «formes" (négative, interrogative, présentative, active, passive) et aux notions de coordination, de juxtaposition et de subordination.

11 Une dizaine d'années plus tard, en 1985, les Instructions pour l'école élémentaire ne font que renforcer cette tendance à donner la priorité à l'analyse, à la reconnaissance des unités, des classes de mots et des fonctions. L'apport de la linguistique se réduit à l'utilisation des manipulations et des transformations, à des fins d'analyse. Les critères de définition sont mis au gout du jour, mais aucun changement notable n'est perceptible en ce qui concerne les objectifs. Dès le cours élémentaire, ces derniers sont en effet définis en des termes qui traduisent bien la volonté de mettre l'accent sur la grammaire réflexive : « distinction des classes de mots », « reconnaissance des fonctions », « repérage des accords». L'ordre dans lequel apparaissent certaines activités est d'ailleurs significatif: "analyse et transformation des formes de phrase», comme si les manipulations venaient compléter la réflexion sur la langue. Les instructions pour le cours moyen ne font que développer les contenus et ne modifient en rien la démarche. Ainsi la notion de groupe ne semble-t-elle être justifiée que par le fait qu'elle permet la décomposition en unités, en l'occurrence en mots. Rien n'est dit sur l'importance du groupe au niveau de la structuration de la phrase, sur son rôle dans le fonctionnement syntaxique ; seule est évoquée la constitution interne des syntagmes :

Le groupe nominal: ses éléments (déterminant, nom, adjectif, complément, proposition relative, apposition).

Le groupe verbal: ses éléments (verbe, compléments directs et indirects, nominaux et pronominaux).

attion est quasiment identique en ce qui concerne le collège. De la même façon que le Plan de rénovation n'était pris que très marginalement en compte dans les textes officiels pour l'école élémentaire, les Programmes et Instructions pour le collège (1985) ne s'inspirent pour ainsi dire pas des propositions élaborées entre temps par la Commission Pierre Emmanuel ou des travaux des groupes de recherche de l'Institut national de recherche et de documentation (INRDP), qui se plaçaient dans la continuité du projet Rouchette. Ici encore, les résistances sont trop fortes pour que se fassent jour des changements majeurs. Particulièrement significatif est le fait que, dans les objectifs généraux, la «communication » n'est pas la finalité essentielle de l'enseignement, mais que c'est avant tout la formation de l'esprit qui justifie la pratique du raisonnement sur la langue :

L'étude raisonnée de la langue et de ses emplois, celle des signes en général,

forment méthodiquement l'esprit et la personne. (p. 24)

ou encore :

Le travail sur la langue apprend à mieux penser. (p. 31)

14

Pratiques, 169-170 | 2016 
« indispensable ", cette recommandation est immédiatement pondérée par le rappel de l'importance de l'analyse :

Il est indispensable de manier la langue dans des situations et des contextes divers [...] Mais la maitrise de la langue se fonde sur une réflexion qui la prend pour objet (morphologie, syntaxe, vocabulaire) et sur une analyse explicite de ses emplois. (p. 25)

On relèvera toutefois une concession à l'esprit de nouveauté, concession qui en reste au niveau des méthodes d'analyse: reprenant une critique formulée dans le Plan de rénovation, le texte de 1985 propose de modeler l'analyse de la phrase complexe sur celle de la phrase simple :

[le professeur] montre que les fonctions syntaxiques peuvent être exercées soit par des mots ou groupes de mots, soit par des propositions subordonnées relatives, complétives ou circonstancielles, réunissant ainsi ce que l'on a appelé l'analyse grammaticale et l'analyse logique. (p. 26)

Il faut également noter la prise en compte, très prudente, de la dimension textuelle et discursive, dont le statut par rapport à la grammaire de phrase n'est guère explicité :

Un principe peut guider la progression: en sixième et cinquième, on étudie la phrase simple et la phrase complexe, afin d'assurer des bases fermes, sans exclure l'étude des suites de phrases qui constituent un texte; en quatrième et en troisième, celle-ci prend la place la plus importante, sans que soient exclus des retours et des compléments à l'étude de la phrase. (p. 27)

17 Il ne s'agit cependant pas, en ce qui concerne la syntaxe, de la didactisation de l'opposition phrase/texte telle qu'elle était pensée dans les travaux scientifiques. C'est ici le simple aspect pédagogique qui est pris en compte, dans la mesure où il est essentiellement question d'éviter l'écueil souvent signalé de la "grammaire pour la grammaire » d'une activité réflexive coupée du contexte de communication :

Une leçon de grammaire ou de vocabulaire ne saurait se dispenser d'une analyse

d'exemples ou de textes, ni du réemploi des éléments étudiés. (p. 28)

En ce qui concerne les contenus, il est bien difficile de trouver les indices d'un changement. La plupart des formulations témoignent toujours de la priorité donnée à l'analyse des unités, à leur décomposition, alors que l'étude des structures, des constructions, n'occupe qu'une place très marginale. Les groupes, les grandes unités sont certes bien présents dans les énumérations des notions, mais ne semblent être mentionnés que comme objets d'un découpage; c'est ainsi qu'une rubrique s'intitule « Le nom et le $\mathrm{GN}$ », comme s'il y avait là deux unités distinctes, en parallèle ; il en va de même avec: "L'adjectif qualificatif et ce qui peut le compléter ». Significatif également, le fait que le premier point proposé sous la rubrique «La phrase complexe» soit l'étude des conjonctions. Cette mise en avant des éléments, de la décomposition des groupes, caractérise également la partie qui traite des fonctions syntaxiques. Il est facile de constater que l'approche de telle ou telle structure, de telle ou telle construction, n'est pas envisagée mais qu'on part en quelque sorte du principe qu'on analyse des faits de langue déjà maitrisés qui ne méritent pas d'être étudiés en tant que tels. Le texte contient certes quelques remarques sur la transitivité, mais le renvoi au dictionnaire montre bien la difficulté qu'il y a à proposer une description, une approche cohérente de la notion. Quant à l'étude des compléments, elle est placée avant celle de l'emploi, transitif ou intransitif, du verbe, ce qui indique bien que la reconnaissance des natures et des fonctions l'emporte sur l'observation des constructions. Si l'on propose l'étude de la 
nature, de la place et du sens des unités, c'est dans un seul but d'identification et non afin de rendre compte d'un fonctionnement d'ensemble.

On constate ainsi une dérive très nette par rapport à ce qu'aurait dû être l'application du distributionalisme, telle que l'entendait le Plan de rénovation ou des auteurs comme $\mathrm{E}$. Genouvrier et J. Peytard que nous avons cités plus haut. Le distributionalisme met certes fondamentalement l'accent sur les unités et la notion de "constituant immédiat » est centrale dans une telle approche, mais la décomposition et la reconnaissance des éléments ne sont en principe que des étapes vers l'étude de la syntaxe; la grammaire générative est un bon exemple de cette méthodologie. Les textes officiels et, à leur suite, les manuels, détournent cette procédure à des fins d'analyse, le changement ne consistant qu'à remplacer, de façon plus ou moins cohérente, une analyse traditionnelle par une analyse modernisée, en s'appuyant sur des critères qui paraissent plus scientifiques pour définir les notions habituelles. Au bout du compte, il n'y a toujours pas de propositions sur l'étude du système syntaxique, sur une approche structurée des constructions: le programme de langue est, encore une fois, le grand absent.

\section{Discours, texte, phrase : les Instructions de 1996}

Un changement de cap assez important caractérise l'esprit des Instructions de 1996, dans lesquelles la priorité est très nettement donnée au discours et au texte, la distinction, souvent reprise par la suite, entre les trois "grammaires", grammaire du discours, grammaire du texte, grammaire de la phrase, semblant résumer la nouvelle organisation de l'étude de la langue. On peut voir dans cet élargissement la méfiance envers une grammaire trop isolée de l'expression, mais aussi l'influence des travaux sur la linguistique du texte et du discours, particulièrement prometteurs à cette époque. Dans un tel cadre, quelle conception de la grammaire de phrase les textes officiels proposentils? L'approche choisie est en partie le reflet des positions théoriques de l'époque : une coupure nette sépare la syntaxe et le domaine du discours et du texte. Seuls quelques faits de langue ont donc droit à un traitement particulier dans la mesure où ils participent au codage de la cohérence textuelle, les autres étant laissés au domaine de la grammaticalité. C'est ainsi que les types et les formes de phrase sont placés sous la rubrique "Discours", en raison des aspects sémantiques et énonciatifs, alors que les classes et les fonctions demeurent du côté de la morphosyntaxe. Une sorte de partage s'établit entre ce qui relève du sens et qui peut dépendre du choix du locuteur et ce qui semble relever de l'arbitraire et des automatismes.

21 La démarche qui accompagne cette conception des trois niveaux est explicitée dans les «Documents d'accompagnement». En ce qui concerne la grammaire de phrase, aucun changement notable ne peut être constaté par rapport aux textes antérieurs. Il n'est toujours pas fait référence aux constructions, aux structures de phrase et la formulation des objectifs fait bien apparaitre que l'important demeure l'identification des unités et des notions :

définir les types et les formes de phrases

identifier et caractériser les principaux constituants de la phrase

préciser les fonctions possibles de certains de ces constituants

Un seul point, qui reste malheureusement trop allusif, aurait pu ouvrir des perspectives intéressantes; il est fait mention des « axes essentiels » que sont « la détermination, la qualification et la complémentation ", qui devraient permettre de regrouper certaines 
notions de la grammaire de phrase. Il est évident que la distinction de ces trois notions ne va pas de soi et que des exemples précis étaient indispensables pour clarifier cette ouverture relativement ambitieuse. L'absence totale, dans les manuels, de toute exploitation de cette piste montre bien qu'une telle suggestion, sans développement ni illustration, ne pouvait que rester lettre morte. Pour le reste, tout se passe comme si la mise en avant de l'« au-delà de la phrase » avait fait passer au second plan toute la partie de la morphosyntaxe qui ne peut être rattachée à la problématique du codage de la cohérence textuelle et discursive, partie qui n'a été l'objet que de quelques réaménagements de détail, sans répercussion fondamentale ni sur les contenus, ni sur la démarche pédagogique.

\section{Le retour à la phrase et au mot : les Instructions de 2008}

Les nombreuses critiques dont ces textes ont été l'objet, critiques qui visaient la notion de "séquence didactique» tout autant, sinon plus, que les contenus d'enseignement, conduisent, avec la rédaction des Instructions de 2008, à un évident retour en arrière. Les principes de ce qu'on peut considérer comme un "retour à la phrase » étaient déjà en germe dans le rapport rédigé en 2006 par A. Bentolila. Ce texte, qui concerne surtout l'école élémentaire mais fait aussi des recommandations pour le collège, peut susciter la curiosité, dans la mesure où il présente une sorte de contradiction entre une idée générale, plusieurs fois exprimée et les conséquences pratiques qui en sont tirées. Le principe central, qui n'avait jamais été développé auparavant et qui remplace en fait les généralités habituelles sur la "communication", est celui de "mise en scène", qui rappelle certaines approches de la grammaire fonctionnelle :

Avant et tout au long de l'apprentissage de la lecture, ces enfants auront été préparés à comprendre qu'au-delà de leur alignement systématique les mots se regroupent pour porter sur la scène du sens des acteurs qui jouent des rôles particuliers. Ils percevront que des décors actualisent cette représentation. Ils réaliseront ainsi que cette grande mise en scène qu'est la compréhension obéit à des indicateurs dont on ne doit jamais ignorer la pertinence. (Bentolila, 2006, p. 13)

et, plus loin :

On a privé les élèves d'une première relation intime avec la grammaire qui seule peut leur permettre de comprendre son rôle essentiel : mettre en scène des acteurs qui se rassemblent dans un cadre spatial et temporel pour faire ensemble quelque chose. (ibid., p. 24)

En fait, on peut penser qu'il s'agit là plutôt d'une façon de retrouver, par le biais de cette métaphore, l'analyse sémantique traditionnelle. Le texte reste d'ailleurs très allusif et rien n'est dit des structures syntaxiques particulières. La "scène du sens" est apparemment remplie d'acteurs et de décors, mais on ne sait rien des relations syntaxe/ sémantique. La simple évocation d'« indicateurs", sans autre précision, ne suffit pas à donner une idée claire du dispositif envisagé. Le rapport ne va pas plus loin. Comment cette "mise en scène", qui, on peut l'imaginer, fait référence au codage des états de choses par les marques linguistiques, se réalise-t-elle dans le système de la langue et, plus généralement, comment envisage-t-on l'interface du plan du contenu et de celui de la forme ? Cette apparente nouveauté théorique n'empêche pas que soient préconisées, une nouvelle fois, la connaissance des unités, leur reconnaissance, leur analyse, qu'il s'agisse des natures ou des fonctions. De façon quelque peu surprenante, la « mise en scène », qui 
doit donner du sens, conduit à privilégier le mot comme unité de base et fondement de la syntaxe :

A l'entrée en cycle 3, les enfants ont besoin de donner du sens à ce qu'ils lisent et doivent pouvoir mettre en mots et en phrases ce qu'ils souhaitent exprimer et transmettre. La reconnaissance des classes de mots (leur nature) est un préalable à tout travail sur la syntaxe. (ibid., p. 14)

ou encore :

La reconnaissance des classes de mots (leur nature), constitue un préalable indispensable à la maîtrise des accords au sein de la phrase et à l'enrichissement de la syntaxe. (ibid., p. 26)

S'instaure ainsi une distance inattendue entre des principes, peu développés, il est vrai, et le retour à l'analyse traditionnelle, ce qui conduit à ne pas s'intéresser la structure des énoncés. Des propositions de ce rapport, le texte des Instructions, en 2008, ne reprend pas l'idée de "mise en scène », mais retient la priorité donnée à l'analyse de la phrase et, surtout, à celle du mot, ce qui conduit par exemple à faire distinguer, en $3^{e}$, les différentes natures de que. La comparaison avec les Instructions antérieures fait apparaitre, à partir de la classe de $4^{\mathrm{e}}$, une rubrique nouvelle, intitulée la « grammaire du verbe ». À y regarder de plus près, on constate que cette dénomination recouvre une fausse nouveauté ; il ne s'agit pas en effet, comme on aurait pu s'y attendre, des constructions du verbe, mais de la forme verbale elle-même, comme l'indiquent bien des formulations comme: "les verbes mis à la forme pronominale », « les verbes essentiellement impersonnels », « les verbes transitifs », etc. Il n'est nullement question de "construction» impersonnelle, pronominale, transitive... Du reste, une bonne partie de cette rubrique est consacrée à l'emploi du subjonctif, ce qui montre bien le flou qui règne autour de cette expression de « grammaire du verbe ».

\section{Les raisons de l'immobilisme}

Au terme de ce survol d'une quarantaine d'années de textes officiels successifs, on peut s'interroger sur les raisons de cet immobilisme, de ce figement. Pourquoi l'étude de la grammaire de phrase n'a-t-elle pas profité des avancées de la recherche théorique dans le domaine de la syntaxe, comme cela s'est produit pour la grammaire de texte dans le domaine de la discursivité ? Revenons rapidement sur chacune des trois étapes que nous venons de décrire. L'appui sur la linguistique structurale, qui caractérise la première période, celle des années 70, n'appelle pas de commentaire particulier, si ce n'est qu'il faut essayer d'expliquer pourquoi, en dépit des recommandations qui allaient dans le sens de la "grammaire pour l'expression », c'est finalement la pratique de l'analyse qui l'a emporté. De la méthode structurale on n'a retenu que les procédures de décomposition de l'analyse distributionnelle, procédures qui, au plan didactique, ne permettent pas, si l'on se limite à leur application, l'étude du fonctionnement syntaxique, et ne peuvent guère conduire qu'à des activités d'analyse. Il faut remarquer aussi que les théories qui se développent alors ne proposent pour ainsi dire pas de réflexion sur la relation syntaxe/ sémantique et ont tendance à séparer nettement les deux domaines. Même lorsqu'il y a, dans des ouvrages pédagogiques comme ceux d'E. Genouvrier, un travail sur les structures de phrase, on en reste au domaine de la grammaticalité, sans que soient établies des relations avec le sens, tant la crainte est grande de voir ressurgir les définitions logicosémantiques. Cette attitude, tout à fait normale au moment où règne 
une syntaxe autonome, explique en partie l'isolement de la grammaire de phrase. C'est à partir des années 90 que le changement aurait pu se produire. On disposait en effet déjà de quelques pistes possibles pour essayer de penser autrement le rôle de la syntaxe et de «donner du sens " à l'approche grammaticale. Certes, les grammaires fonctionnelles étaient encore peu connues et leur application sur le français presque inexistante, mais les travaux de M. Gross et de son école (cf. 1975), le cadre du «lexique grammaire», étaient disponibles et se prêtaient relativement bien à la didactisation. Comme nous l'avons signalé plus haut, la prise en compte de la linguistique du texte s'est opérée à un moment où, au plan théorique, la dichotomie textualité/syntaxe l'emportait nettement sur toute autre conception et on peut presque considérer que c'est le développement, dans le domaine didactique, de l'étude du texte et du discours qui a été la cause, plus ou moins directement, de l'immobilisme qui caractérise la grammaire de phrase. Même lorsque certains concepts sont empruntés à des théories qui ont des approches plus globales du système linguistique, c'est en respectant une séparation stricte des domaines qu'ils sont réutilisés. Il suffit d'observer la façon dont ont été exploitées les notions développées par l'École de Prague, avec la «perspective fonctionnelle de la phrase ». Dans cette théorie, la description d'un énoncé met en jeu trois niveaux étroitement liés : le niveau syntaxique, avec les constructions ; le niveau sémantique, avec les divers rôles; le niveau informationnel, avec les degrés de dynamisme communicatif. Il y avait là, incontestablement, une voie possible pour la didactisation. Or c'est un aspect très restreint de la théorie qui a été isolé; cette organisation d'ensemble a été totalement ignorée et n'a été retenue que la question des enchainements textuels, des diverses progressions thématiques. Alors que le niveau informationnel, fondamental dans le système de la langue, est justifié par le codage syntaxique qu'il met en jeu, les Instructions, les manuels et la pratique scolaire ne vont conserver que ce qui permet de procéder à des analyses stylistiques qui semblent scientifiquement fondées. Avec les textes de 2008, la volonté de rupture avec l'approche discursive est telle que le repli sur la grammaire traditionnelle empêche toute ouverture vers d'autres approches syntaxiques, alors qu'on disposait de synthèses tout à fait utilisables, comme les ouvrages de M. Riegel (1994), de P. Le Goffic (1993) ou de M. Wilmet (1997), manuels qui faisaient d'ailleurs référence pour la préparation aux concours. On ne peut que citer ici une nouvelle fois l'ouvrage Linguistique et enseignement $d u$ français, avec le passage suivant, qui, décrivant la situation dans les années 70, s'applique parfaitement 40 ans plus tard, comme si rien ne s'était passé dans le champ théorique :

Nous voulons seulement signaler que tout se passe comme si, depuis une cinquantaine d'années, l'enseignement grammatical avait ignoré l'effort de la recherche; à l'évidence, il n'a tenu aucun compte du travail de la linguistique générale ou française. (Peytard \& Genouvrier, 1970, p. 87-88)

Indépendamment des modifications - mineures, il est vrai - qui ont pu affecter le contenu des textes officiels, il est frappant de constater comment, tout au long de cette période, se maintient la priorité donnée à l'analyse, à la reconnaissance des catégories, des natures et des fonctions. Les programmes proposés ne sont pas des programmes de langue, dans le sens où ils ne correspondent pas à un apprentissage progressif des structures linguistiques, mais des programmes de grammaire réflexive. Un examen des manuels montrerait d'ailleurs facilement que les activités destinées à faire relever, identifier, distinguer ou nommer l'emportent de loin sur les activités d'expression et de compréhension. 
30 C'est par rapport à ce tableau quelque peu pessimiste que nous voudrions, en guise de conclusion, examiner les nouvelles Instructions qui entreront en vigueur à la rentrée 2016. Dans la mesure où ces textes n'ont pas encore été mis en œuvre, il faut évidemment se garder de porter un jugement définitif et il est nécessaire d'attendre quelque peu avant de pouvoir évaluer les changements qu'ils préconisent. Ceci étant, en se limitant au domaine de la grammaire, on peut relever un certain nombre de points qui s'éloignent nettement de l'approche traditionnelle décrite plus haut et qui apparaissent ainsi comme particulièrement positifs.

31 Au plan méthodologique, on notera que les recommandations qui sont faites concernant les activités d'observation incitent de façon très claire et précise à mettre en œuvre une véritable démarche de découverte qui utilise des corpus dont peuvent faire partie les productions des élèves; on remarquera aussi que cette démarche de découverte doit, plus précisément, s'appuyer sur la problématisation à partir d'un fait de langue, type d'activité qui, notons-le au passage, n'est guère représenté dans les manuels :

Travail à partir des écrits des élèves, acceptables ou non, de corpus de phrases et/ ou de textes créés, de textes littéraires ou non pour inviter à la problématisation (situation-problème). (p. 239)

Il faut également souligner l'attention avec laquelle a été traitée la difficile question des progressions, la tentative de répartir les contenus de l'enseignement grammatical, non seulement du point de vue quantitatif, mais également du point de vue des notions ellesmêmes, les sous-catégories n'étant abordées que peu à peu. Sont ainsi exploitées didactiquement les possibilités de hiérarchisation offertes par le système linguistique.

En ce qui concerne les contenus, la principale nouveauté nous semble résider dans la priorité donnée aux structures syntaxiques et à leur fonctionnement. Les activités d'observation n'ont pas pour objectif premier l'identification des unités mais la découverte des propriétés particulières de telle ou telle structure. L'extrait suivant, parmi bien d'autres, témoigne de ce souci :

Au cycle 4, c'est la syntaxe qui fait l'objet d'une étude plus systématique et c'est dans la perspective de leur fonctionnement syntaxique que sont étudiées les classes de mots et leurs relations. (p. 238)

Ce point de vue, qui rappelle les recommandations du Plan de rénovation, est pour nous très positif et constitue un changement de cap tout à fait notable par rapport aux textes antérieurs. La mise en avant de l'étude des constructions s'accompagne, même si cela demeure peu explicité, d'une certaine conception de la place de la syntaxe, dans la mesure où il est plusieurs fois recommandé que soit prise en considération la relation forme/sens, comme dans les extraits suivants :

Mise en évidence du lien sens-syntaxe. [...] analyse du sens des verbes en fonction de leur construction. (p. 242).

Manipulations syntaxiques pour mettre en évidence les constructions verbales.

Utilisation d'articles de dictionnaires pour relier sens et construction et étudier le champ sémantique des verbes. (ibid.)

35 Il y a là, sans nul doute, une approche nouvelle qui, si elle est considérée comme fondamentale et non comme marginale, devrait modifier en profondeur l'enseignement grammatical.

La lecture de ce texte particulièrement riche - rappelons que nous nous en tenons à la partie grammaticale et que les autres aspects de l'enseignement du français mériteraient bien évidemment aussi une analyse attentive - fait toutefois naitre deux regrets. Le 
premier concerne cette ouverture, que nous venons de mentionner, vers une prise en compte de l'interface sémantique/syntaxe. Il aurait été pertinent, non seulement du point de vue de la cohérence du type de description proposé mais également du point de vue didactique, que cette approche ne se limite pas aux constructions du verbe, mais soit étendue à l'ensemble de la syntaxe. Des Instructions officielles ne sont certes pas un traité de linguistique et ne peuvent proposer une description complète et détaillée des faits de langue. On aurait pu toutefois préciser que les structures de l'énoncé devaient être observées dans le même esprit; des formes de phrase comme la forme impersonnelle, les constructions clivées, les diverses structures à segmentation et à détachement, pour ne citer que quelques exemples, ne sont apparemment pas considérées comme des phénomènes linguistiques relevant de cette démarche, ou, du moins, ne sont pas évoquées dans ce sens. Le renvoi, plus ou moins explicite, de l'étude de ces constructions du côté de la grammaire de texte n'est pas une solution satisfaisante. Si l'on désire donner une certaine cohérence au type de grammaire enseigné et utilisé, la relation construction/sens doit être étendue à l'ensemble de la syntaxe et non pas être restreinte à la question de la transitivité. Sur ces points précis, on ne voit malheureusement guère de progrès par rapport aux programmes antérieurs et la place est laissée à une certaine improvisation, ce qui est pour le moins gênant, étant donné le peu d'informations dont disposent les enseignants dans ce domaine.

Sur un plan plus général, on peut regretter que le statut des activités d'analyse, la part de la « grammaire réflexive » ne soient pas assez clairement définis. Il faut sans doute voir là une certaine hésitation à aller à l'encontre d'habitudes solidement ancrées. Si l'on observe l'organisation de l'étude de la langue au cycle 4 , on constate en effet que trois grandes directions sont proposées :

La grammaire au service des compétences langagières de lecture et d'écriture nécessaires pour s'approprier le sens des textes et mener des analyses littéraires étayées. [...]

La grammaire au service de l'orthographe. [...]

La grammaire au service de la réflexion sur la langue. (p. 238)

Une telle présentation laisse penser que la grammaire réflexive est une activité propre, ayant sa fin en soi. Il est vraiment dommage qu'il n'ait pas été rappelé nettement que l'enseignement grammatical est destiné avant tout à l'apprentissage de la langue, qui ne peut se restreindre au champ de la lecture et de l'écriture. Ceci est sans doute une évidence, mais cette évidence devrait être fortement réaffirmée, afin de montrer comment l'étude de la langue doit être subordonnée à ce but, ce qui justifie alors pleinement les options choisies en ce qui concerne l'étude du fonctionnement du système linguistique, en particulier l'approche fonctionnelle. En l'absence de cette clarification, demeure toujours le risque d'une dérive vers l'«analyse pour l'analyse», qui caractérisait, à des degrés divers, les Instructions antérieures.

On comprend que le type de texte particulier que constituent les Instructions officielles puisse difficilement éviter de composer avec la tradition, d'où une certaine prudence; il n'en reste pas moins que, dans le domaine de la grammaire, ce texte contient bon nombre de propositions novatrices, qui sont en rupture avec l'immobilisme des 40 dernières années et dont on ne peut que se féliciter et les lacunes, les ambigüités, que nous venons de signaler pourraient être prises en compte et clarifiées dans d'éventuels documents d'accompagnement. L'ambition affichée par ce texte court malheureusement le risque d'être freinée par deux difficultés majeures. Se pose en effet la question des outils pédagogiques c'est-à-dire, plus particulièrement, celle des manuels, question qui se 
complique d'ailleurs par le fait que la mise en œuvre de certaines recommandations du texte officiel remet en question l'utilisation même d'un manuel. Quand on voit combien est peu satisfaisante, dans la quasi-totalité des ouvrages scolaires, la didactisation de l'analyse distributionnelle ou des notions de la grammaire de texte, on ne peut qu'être sceptique quant à la possibilité d'élaborer, dans un délai relativement bref, des instruments de travail qui prennent en compte d'une façon cohérente et scientifiquement satisfaisante les nouveaux types d'approche recommandés par ces nouvelles Instructions. Un deuxième obstacle, beaucoup plus difficile à surmonter, sera sans doute constitué par la formation des enseignants, qui, dans leur ensemble, ne sont pas habitués à porter sur les structures linguistiques le regard que préconisent les nouveaux programmes, habitués qu'ils sont, ne serait-ce que par l'influence des manuels, à des activités de reconnaissance et d'analyse des natures et des fonctions. Qu'il s'agisse de formation initiale ou de formation continue, un travail considérable reste à accomplir dans ce domaine. Si rien n'est fait dans ce sens, on peut craindre que les idées et les recommandations novatrices que contiennent les nouvelles Instructions ne demeurent sans lendemain.

\section{BIBLIOGRAPHIE}

BENTOLILA, A., (en collab. avec) DESMARCHELIER, D. \& ORSENNA, E. (2006). Rapport de mission sur

l'enseignement de la grammaire. Paris : Ministère de l'Éducation nationale.

GENOUVRIER, E. \& PEYTARD, J. (1970). Linguistique et enseignement du français. Paris : Larousse.

GROSS, M. (1975). Méthodes en syntaxe. Paris : Hermann.

LE GOFFIC, P. (1993). Grammaire de la phrase française. Paris : Hachette.

RIEGEL, M., PeLlat, J.-C. \& Rioul, R. (1994). Grammaire méthodique du français. Paris : Presses

universitaires de France.

WILMET, M. (1997). Grammaire critique du français. Paris : Hachette/Duculot.

\section{RÉSUMÉS}

Dans cet article, on s'attache à décrire les caractéristiques de l'enseignement de la "grammaire de phrase" dans les textes officiels qui ont été publiés depuis une trentaine d'années. En observant les contenus de la didactique de la syntaxe et les méthodes d'enseignement qui ont été proposés, on tente de définir les facteurs d'un immobilisme qui aboutit, en particulier, à ne pas prendre en compte les données de la linguistique théorique contemporaine. On peut ainsi évaluer les aspects novateurs que présentent les derniers textes officiels qui viennent de paraitre.

In this article, we attempt to describe the characteristics of « sentence grammar » didactics in official texts which were published since around thirty years. By observing the contents of the didactics of the syntax and the teaching methods which were proposed, we try to define the factors of a conservatism which leads, in particular, not to take into account the data of 
contemporary theoretical linguistics. We can so estimate innovative aspects which present the last official texts which have just appeared.

INDEX

Keywords : official Instructions, syllabuses, didactics of language, syntax, sentence grammar, French first language

Mots-clés : instructions officielles, programmes, enseignement de la langue, syntaxe, grammaire de phrase, français langue maternelle

\section{AUTEUR}

\section{BERNARD COMBETTES}

Université de Lorraine, Centre national de la recherche scientifique, Atilf, UMR 7118, F-54000, France 